\title{
Comparison of weight:height ratio and arm circumference in assessment of acute malnutrition
}

\author{
E P CARTER
}

The Department of Haematology, The Hospital for Sick Children, London

SUMMARY Doctors working in famine relief commonly use the weight:height ratio and the circumference of the mid-upper arm to assess the nutritional state of children under 5 . Threshold values indicating moderate and severe malnutrition are usually taken as $80 \%$ and $70 \%$, respectively, of the expected weight:height ratio and arm circumferences of $13.5 \mathrm{~cm}$ and $12.5 \mathrm{~cm}$, respectively. A study of 1260 children aged 1-5 showed that the thresholds of these two variables yielded significantly different proportions of children with malnutrition, the proportion being much larger when arm circumference was used as the criterion. Adjusting the thresholds would result in closer correspondence between the two variables.

When working to relieve famine doctors have to screen large numbers of children as quickly as possible to assess their nutritional state. Two screening mathods are commonly used: the weight:height ratio and measurement of arm circumference. ${ }^{1-6}$ Both measurements are relatively independent of age, sex, and race. Arm circumference is a measure of the lean body mass, whereas the weight:height ratio indicates the extent of acute malnutrition; each, therefore, measures a different aspect of nutritional state. This study assessed the discrepancy that may arise when both measurements are used.

The weight:height ratio expresses a child's weight as the percentage of the expected weight for the child's height. It is usual to accept $80 \%$ of the reference median as the threshold of malnutrition. A ratio of $70-80 \%$ indicates moderate malnutrition and one of less than $70 \%$ indicates severe malnutrition. ${ }^{12}$ Arm circumference increases rapidly in the first year of life $e^{3}$ but then remains fairly constant between the ages of 1 and 5 years. ${ }^{4}$ In well nourished children aged $1-5$ the accepted normal is $16 \mathrm{~cm}$. An arm circumference of $13.5 \mathrm{~cm}$ is commonly accepted as the threshold indicating malnutrition. If the circumference is between 12.5 and $13.5 \mathrm{~cm}$ the child is considered to be moderately malnourished, and measurements below $12.5 \mathrm{~cm}$ indicate severe malnutrition. ${ }^{5-6}$ Thus in any child or population a weight:height ratio of $80 \%$ should be equivalent to an arm circumference of $13.5 \mathrm{~cm}$ and a ratio of $70 \%$ should be equivalent to an arm circumference of $12.5 \mathrm{~cm}$. Feeding centres are usually established in areas where a fifth of children under 5 are malnourished, and any difference between the two indices of malnutrition could lead to difficulty in deciding whether to start relief.

A study was carried out by Lintjørn in 1985 of 115 children in southern Ethiopia, which showed a large difference between the two methods. ${ }^{7}$ We carried out a similar study of a much larger group of children in northern Ethiopia.

\section{Patients and methods}

We carried out a nutritional survey of 1260 children aged 1-5 in the province of Wollo, northern Ethiopia. Weight:height ratio and arm circumference were recorded for each child with Salter scales, wooden length boards, and paper tapemeasures. For the ratio the expected weight for a given height was taken from the median of the World Health Organisation standards. The threshold for moderate malnutrition was taken as a weight: height ratio of less than $80 \%$ and an arm circumference of less than $13.5 \mathrm{~cm}$, and for severe malnutrition the figures were less than $70 \%$ and less than $12.5 \mathrm{~cm}$, respectively.

The $\chi^{2}$ test was used to test the significance of differences between the proportions of children in each nutritional group as assessed by the two measurements.

\section{Results}

Comparison of the weight:height ratios and arm circumferences showed good correlation $(r=0) \cdot 53)$ 


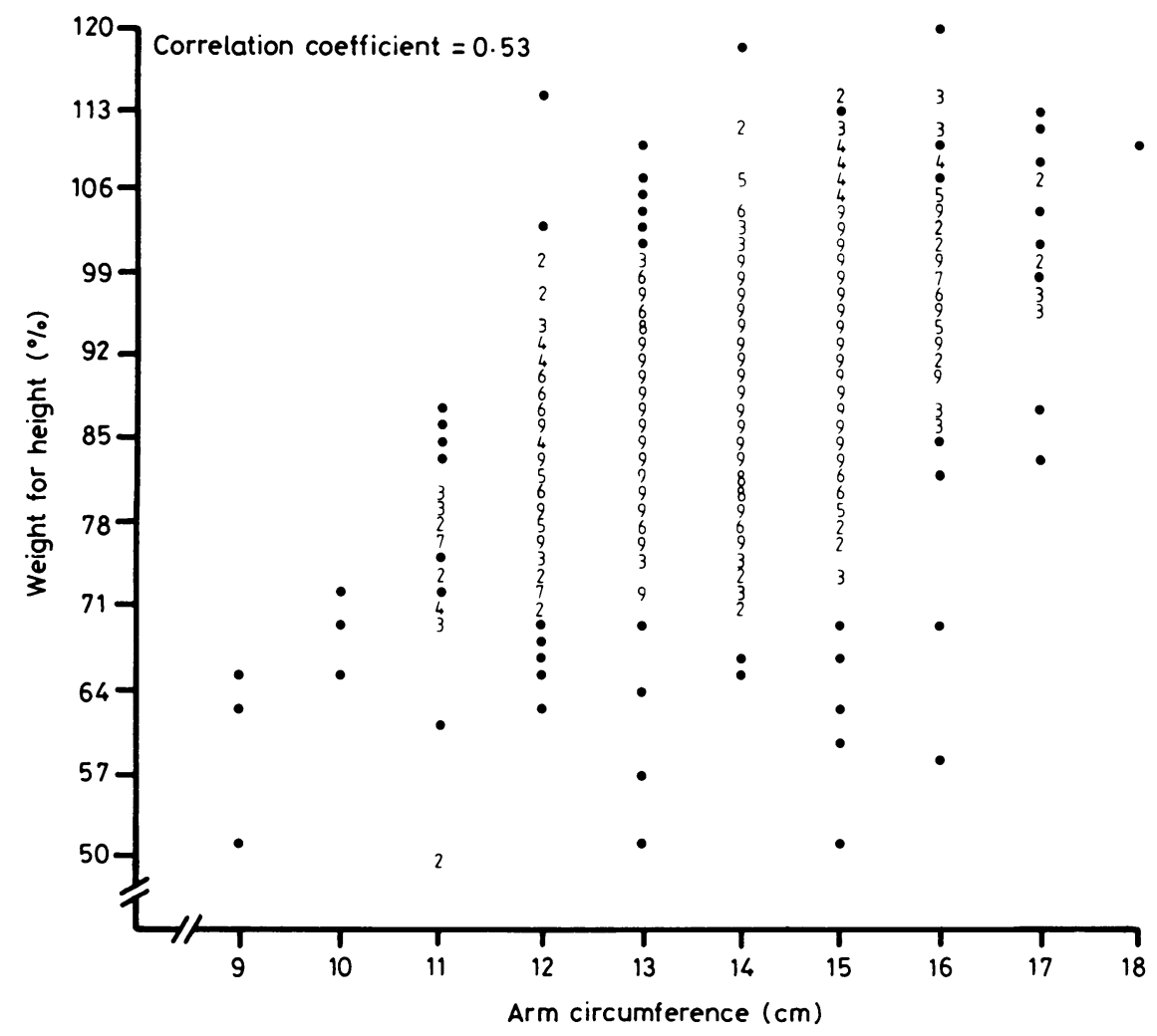

Figure Scattergram showing correlation between weight:height ratio and arm circumference in defining nutritional impairment. Each dot indicates one child, while figures indicate total numbers of children at each point.

between them in defining nutritional impairment (Figure); analysis of variance showed a significant linear trend. The accepted thresholds indicating malnutrition, however, did not correspond. Of the 1260 children, $168(13 \%)$ were diagnosed as malnourished from their weight:height ratio compared with $433(34 \%)$ from their arm circumference. This difference was highly significant $(\mathrm{p}<0 \cdot(001)$.

Table 1 shows the numbers of children classified by the two methods as having moderate and severe malnutrition. The differences between the methods were highly significant $(p<0.0001$ in both cases $)$.

Table 1 Number (percentage) of children in each category of malnutrition

\begin{tabular}{lll}
\hline Malnutrition & Arm circumference & Weight:height ratio \\
\hline Moderate & $264(21)$ & $144(11)$ \\
Severe & $169(13)$ & $24(2)$ \\
\hline Total & $433(34)$ & $168(13)$ \\
\hline
\end{tabular}

Table 2 Diagnostic agreement between weight:height ratios and arm circumference measurements using currently accepted limits

\begin{tabular}{lcccc}
\hline $\begin{array}{l}\text { Arm circumference } \\
(\mathrm{cm})\end{array}$ & Weight:height ratio & & Total (\%) \\
& $<70$ & $70-79$ & $>80$ & \\
\hline$<12 \cdot 5$ & 12 & 63 & 94 & $169(13)$ \\
$12 \cdot 5-1.3 \cdot 5$ & 4 & 40 & 220 & $264(21)$ \\
$>13 \cdot 5$ & 8 & 41 & 778 & $827(66)$ \\
\hline Total $(\%)$ & $24(2)$ & $144(11)$ & $1092(37)$ & $1260(1(6))$ \\
\hline
\end{tabular}

Table 2 presents the figures differently and shows that $830(66 \%)$ of the children were diagnosed as having the same degree of malnutrition by both methods.

\section{Discussion}

This study shows that there is a highly significant linear correlation between the weight:height ratio and arm circumference for all degrees of mal- 
Table 3 Diagnostic agreement between weight:height ratios and arm circumference measurements using recommended limits

\begin{tabular}{llllr}
\hline $\begin{array}{l}\text { Arm circumference } \\
(\mathrm{cm})\end{array}$ & \multicolumn{3}{l}{ Weight:height ratio } & Total (\%) \\
\cline { 2 - 5 } & $<70$ & $70-79$ & $>80$ & \\
\hline$<12 \cdot 5$ & 8 & 22 & 9 & $39(3)$ \\
$12 \cdot 5-13 \cdot 5$ & 4 & 41 & 85 & $130(10)$ \\
$>13 \cdot 5$ & 12 & 81 & 998 & $1091(87)$ \\
\hline Total $(\%)$ & $24(2)$ & $144(11)$ & $1092(87)$ & $1260(100)$ \\
\hline
\end{tabular}

nutrition. We found, however, that the thresholds taken as indicating moderate and severe malnourishment yielded significantly different numbers of children, such that there would be disagreement about whether relief was necessary. The difference may be explained by the fact that the two screening methods measure different aspects of nutritional state, but it is nevertheless important that cut off values correspond.

We recommend that the cut off values be adjusted so that they correspond more closely. If an arm circumference of $12.5 \mathrm{~cm}$ was taken to be equivalent to a weight:height ratio of $80 \%$, and a measurement of $11.5 \mathrm{~cm}$ was taken to be equivalent to $70 \%$, the correlation would be greatly improved, so that $82 \%$ of the values would correspond (Table 3 ) instead of the $66 \%$ correspondence with the currently accepted limits.

I thank Professor D C Morley and Dr Z Ebrahim tor their help in producing this paper.

\section{References}

' Jelliffe DB. The assessment of the nutritional status of the community. Geneva: World Health Organisation, 1966.

2 Oxfam. Practical guide to selective feeding programmes. Oxford: Oxfam Health Unit, 1984.

3 Margo G. Assessment of malnutrition using mid upper arm circumference. Am J Clin Nutr 1977:30:835-7.

${ }^{4}$ Froozani MD. Weight, height and arm circumference in a group of low income Esfahan children. J Trop Pediatr 1978:24:117-9.

5 Shakir A. Morley D. Measuring malnutrition. Lancet 1974:i: 758-9.

' Simmonds S. Vaugh P. Gunn SW. Refugee community health care. Oxford: Oxford Medical Publications. 1983:92-124.

${ }^{7}$ Lintjørn B. Measuring acute malnutrition: a need to re-define cut-off ponts for arm circumference? Lancet 1985;ii:1229-30.

Correspondence to Dr Elaine Carter. Department of Haematology, The Hospital for Sick Children. Great Ormond Street. London WC1N 3JH, England.

Received 17 December 1986 